\title{
Sodium glucose co-transporter-2 inhibitor, Empagliflozin, is associated with significant reduction in weight, body mass index, fasting glucose, and A1c levels in Type 2 diabetic patients with established coronary heart disease: the SUPER GATE study
}

\author{
Satilmis Bilgin ${ }^{1}$. Ozge Kurtkulagi ${ }^{1}$ - Tuba Taslamacioglu Duman ${ }^{1}$ - Burcin Meryem Atak Tel ${ }^{1}$. Gizem Kahveci ${ }^{1}$. \\ Murat Kiran ${ }^{1} \cdot$ Eray Erge $^{1} \cdot$ Gulali Aktas ${ }^{1}$ (1)
}

Received: 14 August 2021 / Accepted: 25 August 2021 / Published online: 2 September 2021

(c) Royal Academy of Medicine in Ireland 2021

\begin{abstract}
Background Empagliflozin, a sodium-glucose co-transporter-2 (SGLT-2) inhibitor, yielded significant beneficiaries in the treatment of type 2 diabetes mellitus (T2DM). It is particularly benefited the diabetic subjects with heart conditions.

Aims We aimed to obtain a real-world data about the effects of empagliflozin add-on treatment on metabolic parameters, cardiovascular risk factors, and anthropometric measures in patients with T2DM.

Methods Type 2 diabetic patients with established coronary heart disease whom empagliflozin added to their treatment were enrolled in the study. Anthropometric measures, clinical and laboratory data, were obtained before and at the 6th month of the empagliflozin treatment. All data before and at the 6th month were compared.

Results Body weight $(p<0.001)$, body mass index $(p<0.001)$, waist $(p<0.001)$ and hip $(p<0.001)$ circumferences, systolic blood pressure $(p=0.006)$, heart rate $(p=0.01)$, LDL cholesterol $(p=0.01)$, fasting plasma glucose $(p<0.001)$, and HbA1c $(p<0.001)$ levels were significantly reduced on 6th month of empagliflozin treatment compared to the baseline values. Estimated GFR $(p=0.66)$, serum creatinine $(p=0.8)$, uric acid $(p=0.40)$, total cholesterol $(p=0.053)$, triglyceride $(p=0.057)$, and HDL $(p=0.09)$ levels were not significantly changed.

Conclusions We suggest that empagliflozin treatment may improve anthropometric measures, metabolic parameters, and blood pressure and does not cause deterioration in kidney functions in type 2 diabetic patients with established coronary heart disease.
\end{abstract}

Keywords Coronary heart disease $\cdot$ Empagliflozin $\cdot$ Fasting glucose $\cdot$ HbA1c $\cdot$ Type 2 diabetes mellitus

\section{Introduction}

Type 2 diabetes mellitus (T2DM) is one of the most common challenges of the physicians in daily clinical practice. Due to the chronic and insidious nature of the disease, it may lead to a number of complications, even unnoticed sometimes. It would be too late to reverse these complications when detected lately. Obstacles in accessing medical care may contribute to the development of diabetic complications, especially in COVID-19 pandemic era that we encounter

Gulali Aktas

draliaktas@yahoo.com

1 Abant Izzet, Baysal University Hospital, Department of Internal Medicine, Bolu, Turkey nowadays. Therefore, effective and potentially less harmful treatment strategies should be preferred in the management of the disease.

Sodium-glucose co-transporter-2 (SGLT-2) inhibitors yield significant contribution to the treatment of the patients with T2DM. A recent meta-analysis concluded that this group of anti-diabetic drugs significantly reduced glycated hemoglobin (HbA1c) levels in the diabetic population [1]. Besides its potent activity in decreasing $\mathrm{HbA} 1 \mathrm{c}$ and contribution to the better metabolic control in T2DM, SGLT-2 inhibitors also decrease pulmonary artery pressure in diabetic subjects suffer from heart failure [2]. It has been proposed as a novel agent that prevents diabetic cardiomyopathy [3]. Especially, it has beneficial effects on cardiovascular health [4-8]. Not only cardiovascular health but also 
renal health is improved with empagliflozin. Authors found that endothelial dysfunction is improved with empagliflozin treatment in diabetic patients with chronic kidney disease [9].

Since SGLT-2 inhibitors, specifically empagliflozin, provided significant cardiovascular beneficial effects and better diabetic regulation, we aimed to observe its effects on the T2DM patients whom treated with empagliflozin in our university clinic. Therefore, we compared anthropometric measures (i.e., weight, body mass index), physical examination findings (such as systolic and diastolic blood pressures), and laboratory parameters of the patients with T2DM at the beginning of the empagliflozin treatment and at those values at 6th month.

\section{Methods}

\section{Design, setting, and population}

After getting approval from the ethics committee (approval number 2021/109), patients with T2DM and coronary heart disease who previously applied to Bolu Abant Izzet Baysal University Department of Internal Medicine were enrolled to the SUPER GATE study, and their data were retrospectively analyzed which obtained from institutional database and patients' files. The subjects whose treatments were controlled, and empagliflozin was added to their treatment for blood sugar regulation, and who had used it for at least 6 months were included in the study. The sample size was determined by power analysis. Initially, 108 subjects were eligible and all enrolled but, of those 24 , were excluded after application of exclusion criteria, and 12 were excluded since they did not show up in the 6th month control visit. The remaining 72 subjects were enrolled to the study. Considering the missing data, 72 patients were included in the study with a 95\% confidence interval (CI) and $20 \%$ variability. Those who used the drug irregularly, who stopped using it, who used it for less than 6 months, and those who discontinued the drug due to side effects were excluded from the study.

\section{Anthropometric and laboratory analyses}

Age, gender, anthropometric measurements (height, weight, waist circumference, hip circumference, body mass index (BMI)), systolic blood pressure, diastolic blood pressure, heart rate of $72 \mathrm{~T} 2 \mathrm{DM}$ patients, glucose, serum creatinine, glycated hemoglobin (HbA1c), estimated glomerular filtration rate (eGFR), aspartate transaminase (AST), alanine transaminase (ALT), c-reactive protein (CRP), serum sodium, potassium, calcium, total cholesterol, low-density lipoprotein (LDL) cholesterol, high-density lipoprotein
(HDL) cholesterol, and triglyceride values were recorded retrospectively. All these parameters were recorded as values before empagliflozin treatment and at the 6th month of treatment. BMI was calculated by division of the weight in $\mathrm{kg}$ to squared height in meters. The effects of this treatment on all these variables were compared.

\section{Statistical analyses}

Statistical analyses were held with SPSS software (SPSS for Windows 20.0, IBM o, Chicago, IL, USA). The average age of the patients was shown as the mean \pm standard deviation. The gender ratio was expressed in numbers and percentages. Study variables before and at the sixth month of empagliflozin treatment were compared with paired samples $T$-test. The $p$ values lower than 0.05 were considered statistically significant.

\section{Results}

The study population consisted of 72 subjects: 56 (77.8\%) men and $16(22.2 \%)$ women. The average age of the study population was $58.7 \pm 8.5$ years. The mean duration of T2DM was $9.5 \pm 7.8$ years in the study population.

The mean weight of subjects at the first examination and at the 6th month control was $83 \pm 13 \mathrm{~kg}$ and $79 \pm 12 \mathrm{~kg}$, respectively. The subjects had an average weight loss of $4 \mathrm{~kg}$ at the 6th month compared to the first visit which was statistically significant $(p<0.001)$. The mean body mass index (BMI) of the subjects at the first visit and the 6th month were $30.1 \pm 3.8 \mathrm{~kg} / \mathrm{m}^{2}$ and $28.8 \pm 3.6 \mathrm{~kg} / \mathrm{m}^{2}$, respectively. BMI of the subjects was decreased by an average of $1.29 \mathrm{~kg} / \mathrm{m}^{2}$, which was statistically significant $(p<0.001)$. The mean waist circumference of subjects at the first examination and the 6th month control was $107.3 \pm 8.9 \mathrm{~cm}$ and $103.2 \pm 3.6 \mathrm{~cm}$, respectively $(p<0.001)$. The mean hip circumference of subjects was reduced from $107.5 \pm 8.8 \mathrm{~cm}$ at the first examination to $103 \pm 9 \mathrm{~cm}$ at the 6th month control visit. The decrease in hip circumference was statistically significant $(p<0.001)$.

The mean systolic blood pressures of subjects at the first examination and at the 6th month control visit were $141 \pm 23 \mathrm{mmHg}$ and $134 \pm 20 \mathrm{mmHg}$, respectively. Systolic blood pressure decreased by an average of $7 \mathrm{mmHg}$ which was statistically significant $(p=0.006)$. Mean heart rates of subjects at the first examination and at the 6th month control were $74 \pm 11$ beats $/ \mathrm{min}$ and $70 \pm 13$ beats/min, respectively. Heart rate decreased by an average of 4 beats per minute which was statistically significant $(p=0.01)$.

There was no statistically significant change in serum sodium $(p=0.39)$, potassium $(p=0.62)$, calcium $(p=0.08)$, CRP $(p=0.93)$, AST $(p=0.07)$, ALT $(p=0.06)$, total 
Table 1 Clinical and the laboratory data of the study population

\begin{tabular}{|c|c|c|c|}
\hline Parameter & $\begin{array}{l}\text { Before empagliflozin } \\
\text { treatment }\end{array}$ & $\begin{array}{l}\text { Sixth month of empagliflozin } \\
\text { treatment }\end{array}$ & $P$ \\
\hline & Mean \pm SD & & \\
\hline Weight $(\mathrm{kg})$ & $83 \pm 13$ & $79 \pm 12$ & $<0.001$ \\
\hline BMI $\left(\mathrm{kg} / \mathrm{m}^{2}\right)$ & $30.1 \pm 3.80$ & $28.6 \pm 3.6$ & $<0.001$ \\
\hline Waist circumference $(\mathrm{cm})$ & $107.3 \pm 8,9$ & $103.2 \pm 3.6$ & $<0.001$ \\
\hline Hip circumference $(\mathrm{cm})$ & $107.5 \pm 8.8$ & $103 \pm 9$ & $<0.001$ \\
\hline Systolic BP (mmHg) & $141 \pm 23$ & $134 \pm 20$ & 0.006 \\
\hline Diastolic BP (mmHg) & $82 \pm 14$ & $79 \pm 11$ & 0.17 \\
\hline Heart rate (per/minute) & $74 \pm 11$ & $70 \pm 13$ & 0.01 \\
\hline Glucose (mg/dL) & $196 \pm 73$ & $149 \pm 54$ & $<0.001$ \\
\hline $\mathrm{HbA} 1 \mathrm{c}(\%)$ & $9 \pm 1.7$ & $7.8 \pm 1.7$ & $<0.001$ \\
\hline Urea $(\mathrm{mg} / \mathrm{dL})$ & $31 \pm 9$ & $34 \pm 10$ & 0.005 \\
\hline Creatinine (mg/dL) & $0.92 \pm 0.1$ & $0.93 \pm 0.1$ & 0.80 \\
\hline eGFR (md/min) & $84.8 \pm 13$ & $84.2 \pm 15$ & 0.66 \\
\hline LDL cholesterol (mg/dL) & $100 \pm 35$ & $91 \pm 34$ & 0.01 \\
\hline $\mathrm{Na}(\mathrm{mmol} / \mathrm{L})$ & $138 \pm 4.5$ & $139 \pm 2.7$ & 0.39 \\
\hline $\mathrm{K}(\mathrm{mmol} / \mathrm{L})$ & $4.6 \pm 0.4$ & $4.6 \pm 0.5$ & 0.62 \\
\hline $\mathrm{Ca}(\mathrm{mg} / \mathrm{dL})$ & $9.1 \pm 0.4$ & $9.2 \pm 0.2$ & 0.08 \\
\hline $\mathrm{CRP}(\mathrm{mg} / \mathrm{L})$ & $8.1 \pm 3.8$ & $7.9 \pm 3.9$ & 0.93 \\
\hline Total cholesterol (mg/dL) & $177 \pm 46$ & $169 \pm 44$ & 0.053 \\
\hline HDL cholesterol (mg/dL) & $42 \pm 12$ & $55 \pm 16$ & 0.09 \\
\hline Triglyceride (mg/dL) & $181 \pm 114$ & $160 \pm 118$ & 0.057 \\
\hline AST & $24 \pm 6$ & $21 \pm 4$ & 0.07 \\
\hline ALT & $27 \pm 8$ & $23 \pm 7$ & 0.06 \\
\hline
\end{tabular}

cholesterol ( $p=0.053)$, triglyceride $(p=0.057)$, and HDL $(p=0.09)$ values of the subjects. Clinical and laboratory parameters of the study population are shown in Table 1 . Mean serum LDL cholesterol level of subjects at the first examination and the 6th month control was $100 \pm 35 \mathrm{mg} / \mathrm{dL}$ and $91 \pm 34 \mathrm{mg} / \mathrm{dL}$, respectively. Subjects had an average decrease of $9 \mathrm{mg} / \mathrm{dL}$ in LDL cholesterol, which was statistically significant $(p=0.01)$.

The mean serum urea level of subjects at the first examination and the 6th month control was $31 \pm 9 \mathrm{mg} / \mathrm{dL}$ and $34 \pm 10 \mathrm{mg} / \mathrm{dL}$, respectively. There was an average increase of $3 \mathrm{mg} / \mathrm{dL}$ in urea values of the subjects, and this increase was statistically significant $(p=0.005)$. However, mean serum creatinine levels of the subjects at the first examination, and at the 6th month control visit were $0.92 \pm 0.1 \mathrm{mg} /$ $\mathrm{dL}$ and $0.93 \pm 0.1 \mathrm{mg} / \mathrm{dL}$, respectively $(p=0.8)$. Similarly, mean eGFR values of the subjects at the first examination and at the 6th month control visit were $84.8 \pm 13 \mathrm{~mL} / \mathrm{min}$ and $84.2 \pm 15 \mathrm{~mL} / \mathrm{min}$, respectively. There was no statistically significant change in eGFR values $(p=0.66)$. Mean fasting glucose levels of subjects at the first examination and at the 6 th month control visit were $196 \pm 73 \mathrm{mg} / \mathrm{dL}$ and $149 \pm 54 \mathrm{mg} / \mathrm{dL}$, respectively. The subjects' glucose level decreased by an average of $47 \mathrm{mg} / \mathrm{dL}$ which was statistically significant $(p<0.001)$. Figure 1 shows the change in the study parameters between the initiation and the 6th month of the empagliflozin treatment.

The mean HbA1c level of subjects at the first examination and the 6th month control was $9 \pm 1.7 \%$ and $7.8 \pm 1.7 \%$, respectively. The subjects' HbA1c level decreased by an average of $1.2 \%$, and this was also statistically significant $(p<0.001)$. Figure 2 shows the change in the HbA1c between before and the 6th month of the empagliflozin treatment.

\section{Discussion}

Tremendous results of the present report, the SUPER GATE study, are evidence of the following: (a) empagliflozin treatment reduced body weight, body mass index, and waist and hip circumferences significantly, which are associated with type 2 diabetes mellitus and cardiovascular disease; (b) systolic blood pressure and heart rate were significantly reduced by 6 months of empagliflozin therapy; (c) empagliflozin treatment caused a significant decrease in fasting plasma glucose, HbA1c, and LDL-cholesterol levels, which are correlated with diabetic complications; and (d) empagliflozin treatment did not cause a significant change in serum 


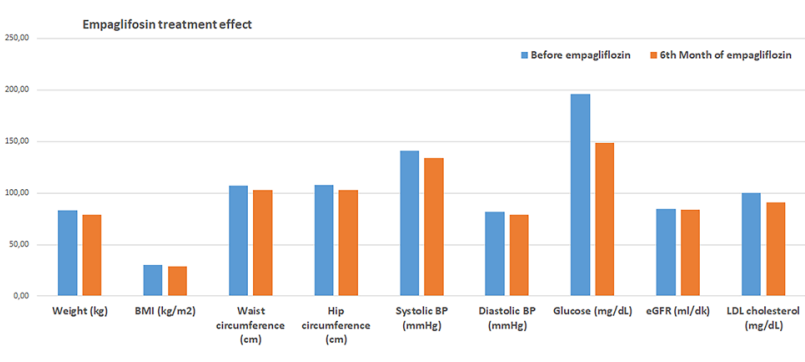

Fig. 1 Change in the study parameters between the initiation and the 6th month of the empagliflozin treatment

creatinine levels and eGFR in type 2 diabetic subjects with coronary heart disease.

Anthropometric measures have been studied in type 2 diabetes mellitus, and it has been established that BMI and waist circumference were associated with the risk of T2DM [10]. These measures also predict the risk of cardiovascular diseases [11]. Therefore, glucose-lowering treatment should be accompanied with measures that reduce body weight, waist circumference, and BMI. The effects of empagliflozin and metformin were compared in subjects with polycystic ovary syndrome, another insulin-resistant state, and authors concluded that empagliflozin caused significant improvements in body weight, waist circumference, hip circumference, and BMI compared to metformin [12]. Treatment duration was 12 weeks, and half of the treatment duration in present study, which was 6 months. Nevertheless, empagliflozin caused 1.4-kg reduction in weight, $1.4-\mathrm{kg} / \mathrm{m}^{2}$ reduction in BMI, 1.6-cm decrease in waist circumference, and $2 \mathrm{~cm}$ of shrinkage in hip circumference [12]. The present study showed that addition of empagliflozin on current

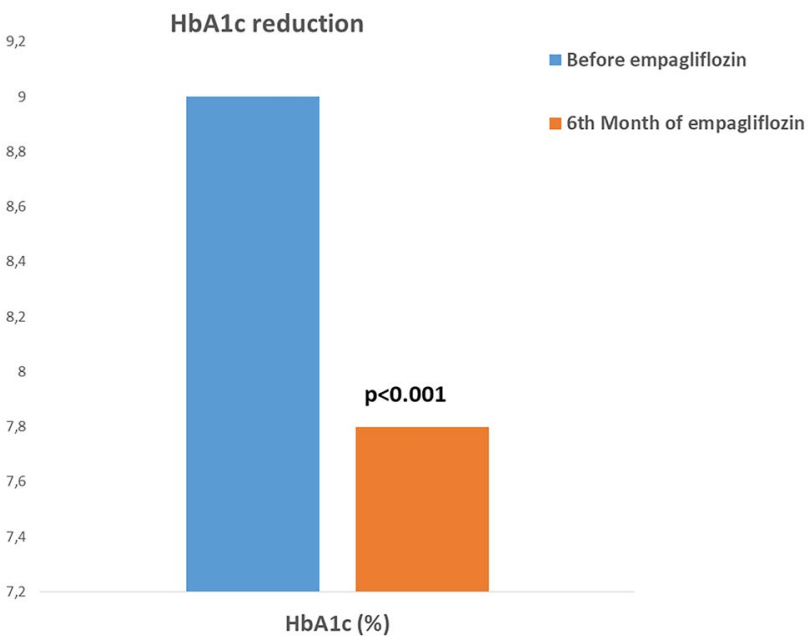

Fig. 2 Change in the HbA1c levels between the initiation and the 6th month of the empagliflozin treatment
T2DM treatment for 6 months provided 3-kg reduction body weight, $1.3-\mathrm{kg} / \mathrm{m}^{2}$ decrease in BMI, $4.3 \mathrm{~cm}$ narrowing in waist circumference, and $2.6 \mathrm{~cm}$ shrinkage of hip circumference. Two other studies were suggested similar reductions in body weight, waist circumference, and other anthropometric measures $[13,14]$. The results of our report were comparable with the data in literature.

In a study by Shin et al., it has been reported that empagliflozin add-on treatment caused significant reductions in both systolic and diastolic blood pressures [13]. The study of Verma et al. suggested their results by reporting significant improvement in systolic and diastolic blood pressures with empagliflozin treatment [15]. Another study was also reported a significant decrease in both systolic and diastolic blood pressures with SGLT-2 treatment [16]. However, we showed a significant decrease in only systolic blood pressure and heart rate in the present study. The reduction in diastolic blood pressure with empagliflozin was $3.4 \mathrm{mmHg}$ which was not statistically significant.

All SGLT-2 inhibitors, including empagliflozin decreased fasting plasma glucose, $\mathrm{HbA1c}$, and improved serum lipid profile [17]. A recent study reported that 12 weeks of SGLT-2 inhibitor (including empagliflozin) treatment was significantly reduced $\mathrm{HbAlc}$ and fasting glucose in the Korean type 2 diabetic population [16]. The authors also found that about $18 \%$ of the subjects reached the HbA1c target which was below $7 \%$ by 12 weeks of SGLT- 2 inhibitor treatment [16]. This rate was $39 \%$ in our study. We also confirmed the previous studies by reporting significant reductions in fasting glucose and $\mathrm{HbA} 1 \mathrm{c}$ by 6 months of treatment of empagliflozin. Rosenstock et al. reported that empagliflozin reduced $\mathrm{HbAlc}$ and fasting glucose significantly when added on to metformin treatment in subjects with type 2 diabetes mellitus [18]. Our results were confirmed the previous studies in literature that showed reductions in metabolic parameters.

These beneficial effects of empagliflozin and other SGLT-2 inhibitor appear to the consequence of improved beta-cell function in diabetic patients [19]. Undesired effects of SGLT-2 inhibitors include urinary tract infection, dehydration, increased urination frequency, and in very rare cases, euglycaemic diabetic ketoacidosis [20]. We did not observe any side effects related to empagliflozin treatment in our study.

In a recent study, authors reported that empagliflozin treatment was associated with deterioration in kidney functions [21]. However, there are also controversial studies in literature. Mortality and morbidity of the diabetic patients with chronic kidney disease were decreased after initiation of empagliflozin treatment [22]. Kadowaki et al. suggested that empagliflozin was improved kidney functions and slowed the rate of decrease in eGFR in the Asian diabetic population received empagliflozin [23]. In present study, the 
change in serum creatinine and eGFR was not statistically different after initiation of empagliflozin.

Possible limitations of our work are the single-center nature of the study and the relatively small study population. However, results of the present study, which is a real-world efficacy study, may add significantly to the current literature.

\section{Conclusion}

We suggest that empagliflozin treatment may improve anthropometric measures, metabolic parameters, and blood pressure and do not cause deterioration in kidney functions in type 2 diabetic patients with established coronary heart disease.

Author contribution Conception and design: SB, GA, and GK. Data collection: SB, MK, EE, and BMAT. Analysis and interpretation of data: TTD, OK, GK, EE, MK, and BMAT. Statistical analysis: GA, SB. Writing the first draft: GK, SB, and OK. Approval of the final manuscript version: SB, GK, TTD, BMAT, OK, EE, MK, and GA. SB and GA take full responsibility for the integrity of the data analysis.

\section{Declarations}

Conflict of interest The authors declare no competing interests.

\section{References}

1. Chen MB, Wang H, Zheng QH et al (2021) Effect of sodiumdependent glucose transporter inhibitors on glycated hemoglobin A1c after 24 weeks in patients with diabetes mellitus: a systematic review and meta-analysis. Medicine (Baltimore) 100(1):e24101. https://doi.org/10.1097/md.0000000000024101

2. Nassif ME, Qintar M, Windsor SL et al (2021) Empagliflozin effects on pulmonary artery pressure in patients with heart failure: results from the EMBRACE-HF Trial. Circulation 143(17):16731686. https://doi.org/10.1161/circulationaha.120.052503

3. Li N, Zhou H (2020) SGLT2 Inhibitors: a novel player in the treatment and prevention of diabetic cardiomyopathy. Drug Des Devel Ther 14:4775-4788. https://doi.org/10.2147/dddt.s269514

4. von Lewinski D, Benedikt M, Tripolt N et al (2021) Can sodium glucose cotransporter 2 (SGLT-2) inhibitors be beneficial in patients with acute myocardial infarction? Kardiol Pol 79(5):503-509

5. Zinman B, Wanner C, Lachin JM et al (2015) Empagliflozin, cardiovascular outcomes, and mortality in type 2 diabetes. N Engl J Med 373(22):2117-2128

6. Mosenzon O, Wiviott SD, Cahn A et al (2019) Effects of dapagliflozin on development and progression of kidney disease in patients with type 2 diabetes: an analysis from the DECLARETIMI 58 randomised trial. Lancet Diabetes Endocrinol 7(8):606-617

7. Packer M, Anker SD, Butler J et al (2020) Cardiovascular and renal outcomes with empagliflozin in heart failure. N Engl J Med 383(15):1413-1424
8. McMurray JJV, DeMets DL, Inzucchi SE et al (2019) The dapagliflozin and prevention of adverse-outcomes in heart failure (DAPAHF) trial: baseline characteristics. Eur J Heart Fail 21(11):14021411. https://doi.org/10.1002/ejhf.1548

9. Dou L, Burtey S (2021) Reversing endothelial dysfunction with empagliflozin to improve cardiomyocyte function in cardiorenal syndrome. Kidney Int 99(5):1062-1064. https://doi.org/10.1016/j. kint.2021.01.008

10. Feller S, Boeing H, Pischon T (2010) Body mass index, waist circumference, and the risk of type 2 diabetes mellitus: implications for routine clinical practice. Dtsch Arztebl Int 107(26):470-476. https://doi.org/10.3238/arztebl.2010.0470

11. Wang Z, Hoy WE (2004) Waist circumference, body mass index, hip circumference and waist-to-hip ratio as predictors of cardiovascular disease in Aboriginal people. Eur J Clin Nutr 58(6):888893. https://doi.org/10.1038/sj.ejcn.1601891

12. Javed Z, Papageorgiou M, Deshmukh H et al (2019) Effects of empagliflozin on metabolic parameters in polycystic ovary syndrome: a randomized controlled study. Clin Endocrinol (Oxf) 90(6):805-813. https://doi.org/10.1111/cen.13968

13. Shin Y, Moon JH, Chin HJ et al (2020) Glycemic efficacy and metabolic consequences of an empagliflozin add-on versus conventional dose-increasing strategy in patients with type 2 diabetes inadequately controlled by metformin and sulfonylurea. Endocrinol Metab (Seoul) 35(2):329-338. https://doi.org/10.3803/ EnM.2020.35.2.329

14. Neeland IJ, McGuire DK, Chilton R et al (2016) Empagliflozin reduces body weight and indices of adipose distribution in patients with type 2 diabetes mellitus. Diab Vasc Dis Res 13(2):119-126. https://doi.org/10.1177/1479164115616901

15. Verma S, Mazer CD, Yan AT et al (2019) Effect of empagliflozin on left ventricular mass in patients with type 2 diabetes mellitus and coronary artery disease: the EMPA-HEART CardioLink-6 randomized clinical trial. Circulation 140(21):1693-1702. https:// doi.org/10.1161/circulationaha.119.042375

16. Hong AR, Koo BK, Kim SW et al (2019) Efficacy and safety of sodium-glucose cotransporter-2 inhibitors in Korean patients with type 2 diabetes mellitus in real-world clinical practice. Diabetes Metab J 43(5):590-606. https://doi.org/10.4093/dmj.2018.0134

17. Zaccardi F, Webb DR, Htike ZZ et al (2016) Efficacy and safety of sodium-glucose co-transporter-2 inhibitors in type 2 diabetes mellitus: systematic review and network meta-analysis. Diabetes Obes Metab 18(8):783-794. https://doi.org/10.1111/dom.12670

18. Rosenstock J, Seman LJ, Jelaska A et al (2013) Efficacy and safety of empagliflozin, a sodium glucose cotransporter 2 (SGLT2) inhibitor, as add-on to metformin in type 2 diabetes with mild hyperglycaemia. Diabetes Obes Metab 15(12):1154-1160. https:// doi.org/10.1111/dom.12185

19. Ferrannini E, Muscelli E, Frascerra S et al (2014) Metabolic response to sodium-glucose cotransporter 2 inhibition in type 2 diabetic patients. J Clin Invest 124(2):499-508. https://doi.org/10. 1172/jci72227

20. Lupsa BC, Inzucchi SE (2018) Use of SGLT2 inhibitors in type 2 diabetes: weighing the risks and benefits. Diabetologia 61(10):2118-2125. https://doi.org/10.1007/s00125-018-4663-6

21. Wanner C, Inzucchi SE, Lachin JM et al (2016) Empagliflozin and progression of kidney disease in type 2 diabetes. N Engl J Med 375(4):323-334. https://doi.org/10.1056/NEJMoa1515920

22. Wanner C, Lachin JM, Inzucchi SE et al (2018) Empagliflozin and clinical outcomes in patients with type 2 diabetes mellitus, established cardiovascular disease, and chronic kidney disease. Circulation 137(2):119-129. https://doi.org/10.1161/circulationaha.117. 028268

23. Kadowaki T, Nangaku M, Hantel S et al (2019) Empagliflozin and kidney outcomes in Asian patients with type 2 diabetes and established cardiovascular disease: results from the EMPA-REG 
OUTCOME(®) trial. J Diabetes Investig 10(3):760-770. https:// doi.org/10.1111/jdi.12971
Publisher's Note Springer Nature remains neutral with regard to jurisdictional claims in published maps and institutional affiliations. 